\title{
Role of ferroptosis induced by a high concentration of calcium oxalate in the formation and development of urolithiasis
}

\author{
ZIQI HE*, WENBIAO LIAO*, QIANLIN SONG* , BIN LI, JUNWEI LIU, \\ YUNHE XIONG, CHAO SONG and SIXING YANG
}

Department of Urology, Renmin Hospital of Wuhan University, Wuhan, Hubei 430060, P.R. China

Received July 5, 2020; Accepted October 6, 2020

DOI: $10.3892 / \mathrm{ijmm} .2020 .4770$

\begin{abstract}
Ferroptosis is an iron-dependent lipid peroxidation process. Although the involvement of ferroptosis in kidney diseases has recently been reported, the association between ferroptosis and urolithiasis remains unclear. The present study examined the effects of ferroptosis on calcium oxalate (CaOx) crystal-induced renal tubular epithelial cell injury in vivo and in vitro. First, renal tubular epithelial cells were exposed to various concentrations of $\mathrm{CaOx}$. By measuring cell viability, $\mathrm{Fe}^{2+}$ levels, lipid peroxidation levels and the levels of ferroptosis-related proteins, it was identified that the relative expression of the ferroptosis agonist proteins, p53, long-chain acyl-CoA synthetases (ACSL4), transferrin (TF) and transferrin receptor (TRC), increased, while the relative expression of the ferroptosis inhibitory proteins, solute carrier family 7 member 11 (SLC7A11, XCT) and glutathione peroxidase 4 (GPX4), decreased significantly. Furthermore, the levels of $\mathrm{Fe}^{2+}$ and lipid peroxidation gradually increased, while cell viability significantly decreased. From these results, it was noted that the extent of $\mathrm{CaOx}$-induced ferroptosis activation and cell injury was dependent on the $\mathrm{CaOx}$ concentration. To further investigate the association between ferroptosis and renal tubular epithelial cell injury, the ferroptosis agonist, erastin, and the ferroptosis inhibitor, ferrostatin-1, were used to regulate the degree of ferroptosis at the same $\mathrm{CaOx}$ concentration in in vivo and in vitro experiments. $\mathrm{CaOx}$-induced ferroptosis and damage to renal tubular epithelial cells and renal tissue were investigated. Finally, it was identified that through the regulation of ferroptosis levels, renal tubular epithelial cell injury increased significantly when the ferroptosis level increased, and vice versa. On the whole, the present
\end{abstract}

Correspondence to: Professor Sixing Yang, Department of Urology, Renmin Hospital of Wuhan University, 238 Jiefang Road, Wuchang, Wuhan, Hubei 430060, P.R. China

E-mail: sxyang2004@163.com

*Contributed equally

Key words: ferroptosis, urolithiasis, HK-2 cells, calcium oxalate, cell injury results indicated that ferroptosis is essential for renal tubular epithelial cell injury induced by $\mathrm{CaOx}$ crystals. This finding is highly significant and promotes the further investigation of the association between ferroptosis and urolithiasis.

\section{Introduction}

Urolithiasis is a type of solid mass that occurs in all parts of the urinary system. It is a disease with a complex etiology and is associated with a high incidence and recurrence rates (1). In recent years, the incidence of urolithiasis has increased each year, thereby placing a heavy burden on the global healthcare system (2). The formation of urinary calculi is a complex process, which includes urinary supersaturation, crystal nucleation, crystal growth and aggregation, and crystal attachment to renal tubular epithelial cells (3). The influence of various factors leads to the difference in the composition of calculi in patients with urinary calculi, among which the proportion of calcium oxalate stones and the mixed stone of calcium oxalate and calcium phosphate stones accounts for approximately $70 \%$. Calcium oxalate $(\mathrm{CaOx})$ is an important component of oxalate calculi, and the supersaturated state of $\mathrm{CaOx}$ in urine is a major risk factor for urolithiasis (4). This aspect prompted us to conduct research on $\mathrm{CaOx}$-induced development of urolithiasis.

Ferroptosis is a novel type of regulatory cell death caused by iron-dependent lipid peroxidation (5). Lipid peroxidation occurs due to the depletion of glutathione in cells. Moreover, $\mathrm{Fe}^{2+}$ is transported at an increased rate in cells. A combined effect of these two factors causes the accumulation of reactive oxygen species (ROS) in cells, eventually leading to ferroptosis. p53 is a classic tumor suppressor gene (6). XCT is a light chain subunit of the cystine/glutamate transport system, which can promote the uptake of cystine and the biosynthesis of glutathione, and can produce a variety of antioxidants in cells, thus protecting the cells from oxidative stress (7). Glutathione peroxidase 4 (GPX4) is a subtype of glutathione peroxidase and the only one that can detoxify lipid peroxidase $(8,9)$. During ferroptosis, p53 induces a decrease in the transcription efficiency of XCT via a transcription-dependent mechanism, which leads to a decrease in intracellular cystine intake and a corresponding decrease in the content of synthetic cysteine. As the rate-limiting precursor of GSH synthesis, the decrease in cysteine level directly leads to a decrease in GSH synthesis 
level, which indirectly affects the activity level of GPX4 in cells and finally leads to lipid peroxidation (6-9). Long-chain acyl-CoA synthetases (ACSL4) is a member of the Acyl-CoA synthetase long-chain family (10), which can esterify arachidonoyl and adrenoyl into phosphatidylethanolamine in cells. Phosphatidylethanolamine, as the only esterified oxygenation center in endoplasmic reticulum (ER) septum, plays an important role in ferroptosis (11). The overload of $\mathrm{Fe}^{2+}$ in cells is also a necessary factor for the induction of ferroptosis. $\mathrm{Fe}^{3+}$ can combine with transferrin (TF) in plasma. TF then combines with transferrin receptor (TRC) on the cell membrane, carrying $\mathrm{Fe}^{3+}$, which is ingested by the receptor cells in the form of endocytosis. The TRC-TF complex entering the cell is acidified, which alters the conformation of the TF-TRC complex protein and promotes the release of iron. Finally, the released $\mathrm{Fe}^{3+}$ is reduced to $\mathrm{Fe}^{2+}$ by ferrireductases of the STEAP protein family $(12,13)$.

According to previous studies, $\mathrm{CaOx}$ crystal-induced autophagy, endoplasmic reticulum (ER) stress (ERS) and epithelial-mesenchymal transition (EMT) play important roles in the formation of urolithiasis (14-17). Previous research has also confirmed that ferroptosis is closely associated with autophagy, ERS and EMT (18-20). It is understood that ferroptosis is caused by a type of $\mathrm{Fe}^{2+}$ overload-dependent lipid ROS. Related studies have reported that the exposure of renal tubular epithelial cells to high concentrations of $\mathrm{CaOx}$ crystals can produce excessive ROS, leading to inflammation and injury to the surrounding tissue (21). Thus, it can be considered that ferroptosis is associated with the development of urolithiasis. Furthermore, during the formation of urolithiasis, renal tubular epithelial cells and renal tissue can be injured by autophagy, which is induced by ROS. Bound iron is stable in cells and does not affect their normal physiological function. Autophagy can degrade iron storage proteins in cells, leading to excessive release of free iron and accumulation of ROS, thereby activating the process of ferroptosis (12). These research findings indicate that ferroptosis is inextricably linked to a variety of causative factors for urolithiasis, which provides further reason to suggest the aforementioned scientific hypothesis. However, with regard to the current research status, although ferroptosis has been reported to be a causative factor of renal diseases, its role in the development of urolithiasis is unclear $(18,22)$. Therefore, as ferroptosis is considered to be closely associated with multiple causes of urolithiasis, the role of ferroptosis in the formation of urolithiasis needs to be understood. The present study preliminarily explored the role of ferroptosis in the development of urolithiasis.

\section{Materials and methods}

Main reagents and antibodies. $\mathrm{CaOx}$ (cat. no. 455997-5G) was purchased from Sigma-Aldrich; Merck KGaA. Erastin (cat. no. S7242) and Ferrostain-1 (cat. no. S7243) were ordered from Selleck Chemicals Co., Ltd. The CCK-8 kit (cat. no. CK04) was obtained from Dojindo Laboratories Co., Ltd. DAPI (cat. no. P0131), the mitochondrial membrane potential assay kit (cat. no. C2006) and the TUNEL staining kit (cat. no. C1086) were purchased from Beyotime Institute of Biotechnology, Inc. The lactate dehydrogenase (LDH) assay kit (cat. no. A020-2-2), the reduced glutathione (GSH) assay kit (cat. no. A006-2-1), the superoxide dismutase (SOD) assay kit (cat. no. A001-3-2), the total antioxidant capacity (T-AOC) assay kit (cat. no. A015-2-1) and the cell malondialdehyde (MDA) assay kit (cat. no. A003-4-1) were purchased from Nanjing Jiancheng Bioengineering Institute Co., Ltd. The MitoSOX Red Mitochondrial Superoxide Indicator (cat. no. 40778ES50) was obtained from Yeasen Biotech Co., Ltd. The catalase activity assay kit (cat. no. BC0205) and penicillin-streptomycin solution (cat. no. P1400) were obtained from Beijing Solarbio Science \& Technology Co., Ltd. The neutrophil gelatinase-associated lipocalin (NGAL; cat. no. CSB-E09409r) and kidney injury molecule-1 (KIM-1; cat. no. CSB-E08808r) ELISA kits were purchased from Cusabio Technology, LLC. Anti-GAPDH (cat. no. 10494-1-AP), anti-p53 (cat. no. 10442-1-AP), anti-GPX4 (cat. no. 14432-1-AP), anti-TF (cat. no. 17435-1-AP) and anti-TRC (cat. no. 1004-2-AP) antibodies were ordered from Proteintech Group, Inc. The iron assay kit (cat. no. ab83366), and anti-XCT (cat. no. ab37185) and anti-ACSL4 (cat. no. ab155282) antibodies were obtained from Abcam. DMEM/F12 (cat. no. C10010500BT) and fetal bovine serum (cat. no. 10270-106) were purchased from Thermo Fisher Scientific, Inc.

Cells, cell culture and exposure. Human proximal tubular cells (HK-2 cells) were obtained from the Cell Bank of the Chinese Academy of Sciences, maintained in complete DMEM/F12 (supplemented with 10\% fetal bovine serum and $100 \mathrm{U} / \mathrm{ml}$ penicillin/streptomycin) and cultured at $37^{\circ} \mathrm{C}$ in an incubator with $5 \% \mathrm{CO}_{2}$. $\mathrm{CaOx}$ crystals were added to the serum-free DMEM/F12 to prepare the $\mathrm{CaOx}$ intervention solution. When cell confluence reached $80 \%$, the cells were exposed to 0 (negative control; NC), 1, 2 and $4 \mathrm{mM} \mathrm{CaOx}$ intervention solution for $24 \mathrm{~h}$ prior to analysis. The CCK-8, LDH and MDA assays were then performed, and their results were obtained using a microplate reader, as described below. Morphological changes in the cells were observed using an inverted microscope, and the protein levels of p53, XCT, ACSL4, GPX4, TF and TRC were examined by western blot analysis. Subsequently, the HK-2 cells were treated with erastin (agonist of ferroptosis, $10 \mu \mathrm{M}$ ) and ferrostatin-1 (inhibitor of ferroptosis, $8 \mu \mathrm{M}$ ) with or without $\mathrm{CaOx}$ intervention solution $(2 \mathrm{mM})$ for $24 \mathrm{~h}$.

Animal experiments. All animal experiment protocols were approved by the Animal Care Committee of Wuhan University (Wuhan, China) and the Laboratory Animal Welfare and Ethics Committee of Renmin Hospital of Wuhan University. A total of 32 male adult Sprague-Dawley rats (weight, 180-220 g; age, 6 weeks) were used in the experiments. Rat models of urolithiasis were established by the administration of $0.75 \%$ ethylene glycol (EG) in the drinking water for 4 weeks. The rats were randomly divided into 4 groups ( $\mathrm{n}=8$ per group) as follows: i) The NC group; ii) urolithiasis model group; iii) nephrolithiasis model treated with erastin $(10 \mathrm{mg} / \mathrm{kg} / \mathrm{day})$ group; and iv) nephrolithiasis model treated with ferrostatin-1 ( $4 \mathrm{mg} / \mathrm{kg} /$ day) group. In the latter 2 groups, the rats were treated with erastin and ferrostatin-1, respectively, by an intraperitoneal injection.

Cell morphology. HK-2 cells were exposed to 0,1,2 and $4 \mathrm{mM}$ $\mathrm{CaOx}$ for $24 \mathrm{~h}$. Morphological changes and cell confluence 
were then observed using an inverted microscope (IX51; Olympus Corporation) at x 200 magnification.

Cell activity assay. Cell activity was measured using a CCK-8 assay kit. The appropriate amount of CCK- 8 reagent was added to the cells in strict accordance with the manufacturer's instructions. After complete mixing, the cells were placed in an incubator $\left(37^{\circ} \mathrm{C}, 5 \% \mathrm{CO}_{2}\right)$ for $2.5 \mathrm{~h}$. The optical density (OD) value was measured at $450 \mathrm{~nm}$ using a microplate reader (VICTOR Nivo; PerkinElmer Corporation).

LDH analysis. LDH is a very stable enzyme involved in energy metabolism of the body. The change in the quality and quantity of LDH directly affects the energy metabolism of the body. A pathological state of tissues and organs leads to the release of LDH into the blood (23). In the present study, the LDH levels were measured using an LDH assay kit. The supernatant of the cell culture medium of each group was collected, and subsequent analyses were performed in strict accordance with the requirements of the supplier's manual. The OD value was measured at $450 \mathrm{~nm}$ using a microplate reader.

Cell biochemistry assay. GSH, CAT, T-AOC, SOD and MDA are classic indicators for measuring the capacity of cells to resist antioxidant and oxidative stress (24-27). In the present study, the levels of GSH were measured using the reduced glutathione assay kit. The levels of CAT were measured using the catalase activity assay kit. The degrees of T-AOC were detected using the total antioxidant capacity assay kit. The levels of SOD were measured using the superoxide dismutase assay kit. The levels of lipid peroxidation were measured using the cell malondialdehyde assay kit. Following treatment, the cells were homogenized (reagents provided with the kits) and centrifuged $\left(15,00 \mathrm{~g} \mathrm{x} 10 \mathrm{~min}, 25^{\circ} \mathrm{C}\right)$ to remove the precipitate. The supernatant was collected and the assay kit reagents were added, and the assays were performed in strict accordance with the respective manufacturer's instructions. The OD value was measured using a microplate reader.

Mitochondrial injury assay. Changes in mitochondrial membrane potential reflect mitochondrial injury. In the present study, the degree of mitochondrial injury was determined using the mitochondrial membrane potential assay kit with JC-1. All reagents were prepared in advance according to the instructions. Following treatment, the supernatant was removed, and HK-2 cells were washed once with PBS; the cells were then collected in an Eppendorf tube. In accordance with the manufacturer's instructions, an appropriate amount $(1 \mathrm{ml})$ of JC-1 staining reagent was added, and the cells were incubated for $20 \mathrm{~min}$ at $37^{\circ} \mathrm{C}$ in $5 \% \mathrm{CO}_{2}$. Following incubation [HBSS medium (containing $\mathrm{Ca}^{2+}, \mathrm{Mg}^{2+}$ ) was used to prepare a $5 \mu \mathrm{M}$ probe working solution; an appropriate amount of probe working solution was then added and incubated at $37^{\circ} \mathrm{C}$ for $10 \mathrm{~min}$ in the dark], the cells were washed with JC-1 buffer and centrifuged at $600 \mathrm{x} \mathrm{g}$ and at $4^{\circ} \mathrm{C}$ for $3 \mathrm{~min}$. This process was repeated twice. Finally, the cells were resuspended in JC-1 buffer for flow cytometric analysis by using a flow cytometer (BD FACSCalibur, BD Biosciences). Flow cytometry was performed at an excitation wavelength of $488 \mathrm{~nm}$, an emission wavelength FL1 $(E M=525 \pm 20 \mathrm{~nm})$, FL2 $(\mathrm{EM}=585 \pm 20 \mathrm{~nm})$.
FlowJo (8.8.2, BD Biosciences) software was used to analyze the data.

ROS analysis. The levels of ROS were detected using the MitoSOX Red Mitochondrial Superoxide Indicator. Once the probe enters the mitochondria, it can be oxidized by superoxide to produce red fluorescence. HBSS medium (containing $\mathrm{Ca}^{2+}$, $\mathrm{Mg}^{2+}$ ) was used to prepare a $5 \mu \mathrm{M}$ probe working solution; an appropriate amount of probe working solution was then added and incubated at $37^{\circ} \mathrm{C}$ for $10 \mathrm{~min}$ in the dark. Following incubation, the working solution was removed, and the cells were washed twice with buffer solution. Finally, the outbreak degree of ROS in each group was observed with an inverted fluorescence microscope (IX1; Olympus Corporation) at x200 magnification.

Western blot analysis. Both HK-2 cells and rat kidney tissues were completely lysed using cold RIPA buffer, and proteins were extracted from the lysed cell/tissue suspension. Protein was quantified by BCA assay (cat. no. PC0020, Beijing Solarbio Science \& Technology Co., Ltd), and the same amount (30 $\mu \mathrm{g})$ of proteins were separated using 10-12\% SDS-PAGE gel and then transferred to PVDF $(0.45-\mu \mathrm{M})$ membranes. Subsequently, the PVDF membranes was blocked at room temperature using $5 \%$ skim milk. The membranes were incubated with $\mathrm{p} 53, \mathrm{XCT}$, GPX4, ACSL4, TF and TRC antibodies $(1: 1,000)$ overnight at $4^{\circ} \mathrm{C}$. The membranes were then washed with TBST buffer and incubated with anti-rabbit $\operatorname{IgG}(\mathrm{H}+\mathrm{L})$ secondary antibodies (cat. no. 5366S, Cell Signaling Technology, Inc.; 1:20,000) for $1 \mathrm{~h}$ at room temperature. Finally, the relative expression levels of each protein were observed by an Odyssey dual color infrared laser imager (Odyssey Infrared Imaging System, LI-COR Corporation), and data were analyzed using Odyssey software (Odyssey Version 3.0.29, LI-COR Corporation).

Immunofluorescence assay. The HK-2 cells were seeded on coverslips; following treatment as described above, the medium was removed, and HK-2 cells were washed twice with cold PBS. The cells were then fixed with $4 \%$ paraformaldehyde (cat. no. P0099; Beyotime Institute of Biotechnology, Inc.) for $15 \mathrm{~min}$ and washed twice with cold PBS. The cells were blocked with 5\% BSA (cat. no. ST023; Beyotime Institute of Biotechnology, Inc.) at room temperature and washed twice with cold PBS. The cells on coverslips were incubated with XCT, GPx4, ACS14, TF and TRC antibodies $(1: 100)$ at $4^{\circ} \mathrm{C}$ for $24 \mathrm{~h}$. This was followed by incubation with FITC- or Cy3-conjugated secondary antibodies (Servicebio; cat. nos. GB22303 and GB21303, respectively) at room temperature for $1 \mathrm{~h}$. Finally, the coverslips were blocked with the antifade mounting medium with DAPI. The fluorescence intensity was measured using an automatic fluorescence microscope (BX51; Olympus Corporation) at x200 magnification.

Histological analysis. The degrees of renal histological injury were determined by Von Kossa staining and the renal tubule histopathological score. After 4 weeks of treatment, the rats were exposed to carbon dioxide ( $30 \%$ of $\mathrm{CO}_{2}$ ) and sacrificed by decapitation, then their kidneys were then removed, fixed with $4 \%$ paraformaldehyde and embedded in paraffin. The tissue paraffin block was sliced and the slices were immersed 
in xylene for $30 \mathrm{~min}$, followed by dewaxing in an ethanol gradient solution. A silver nitrate solution was then dropped on the slices and irradiated under an ultraviolet lamp for $10 \mathrm{~min}$. The excess silver nitrate solution was washed away with distilled water, a hyposulfite solution was added to the slices, and the slices were placed at room temperature for $2 \mathrm{~min}$. Finally, the cell nuclei were counterstained (hematoxylin, $10 \mathrm{~min}, 25^{\circ} \mathrm{C}$; eosin, $40 \mathrm{sec}, 25^{\circ} \mathrm{C}$ ) with an $\mathrm{H} \& \mathrm{E}$ staining solution (cat. no. G1120, Beijing Solarbio Science \& Technology Co., Ltd.). Scoring standards for the degree of renal tubule injury were as follows: 1 point for marked renal tubular expansion and flattening of cells; 1 point for brush border injury and 2 points for brush border loss; 2 points for cellular cast formation, and 1 point for necrotic cells and cell desquamation (non-casted formation or cell debris). All slices were observed under an automatic microscope (BX51; Olympus Corporation) at x400 magnification.

In addition, following treatment, venous blood samples and 24-h urine samples of rats were collected. The blood samples were placed in an automatic clinical chemistry analyzer to detect serum creatinine (CRE) and blood urea nitrogen (BUN) levels. The 24-h urine samples were subjected to NGAL and KIM-1 analyses using the NGAL and KIM-1 ELISA assay kits. Samples and kits were used in strict accordance with the manufacturer's instructions, and the NGAL and KIM-1 levels were determined using a microplate reader (VICTOR Nivo; PerkinElmer Corporation).

TUNEL assay. The preparation of cell coverslips and tissue slices was consistent with that of the previous experiment. After the tissue slices were prepared, proteinase $\mathrm{K}$ was used to strengthen the binding between the kit probe and the broken DNA. The samples were then mixed with the TUNEL staining kit reagent and incubated at $37^{\circ} \mathrm{C}$ for $1 \mathrm{~h}$. Finally, the samples were blocked with the antifade mounting medium with DAPI. The positive rate of TUNEL staining was observed using an automatic fluorescence microscope (BX51; Olympus Corporation) at x200 magnification.

$\mathrm{Fe}^{2+}$ assay. The levels of intracellular $\mathrm{Fe}^{2+}$ were detected using the iron assay kit. Following treatment, both HK-2 cells and rat kidney tissues were homogenized in an appropriate amount of iron assay buffer. The samples and kits were used in strict accordance with the manufacturer's instructions, and the absorbance was measured at $593 \mathrm{~nm}$ using a microplate reader.

Statistical analysis. All data from the experiments are expressed as the means \pm standard error of the mean of $\geq 3$ independent experiments. All statistical analyses were performed using GraphPad Prism software version 6.0 (GraphPad Software, Inc.). Significant differences among multiple groups were analyzed with one-way analysis of variance followed by the Bonferroni's post hoc test. $\mathrm{P}<0.05$ was considered to indicate a statistically significant difference.

\section{Results}

CaOx activates ferroptosis in HK-2 cells in a concentration-dependent manner. With the increase in the concentration of $\mathrm{CaOx}$, the morphology of the HK-2 cells gradually changed from an original oval shape to a long spindle-like shape, and the cell confluency decreased (Fig. 1A). To examine the role of $\mathrm{CaOx}$-mediated ferroptosis in the formation of urolithiasis, the relative expression levels of ferroptosis-related proteins were detected in the $\mathrm{HK}-2$ cells following exposure to $\mathrm{CaOx}$. It was identified that with the increase in the $\mathrm{CaOx}$ concentration, compared with the $\mathrm{NC}$ group, the relative expression of the ferroptosis agonist proteins, p53, ACSL4, TF and TRC, increased, while that of the ferroptosis inhibitory proteins, XCT and GPX4, significantly decreased (Fig. 1B and C). Changes in the MDA level reflect the changes in intracellular lipid peroxidation levels; compared with the NC group, the MDA levels were significantly increased in the treatment groups (Fig. 1D, left panel). In terms of changes in protein expression and lipid peroxidation, $\mathrm{CaOx}$ activated ferroptosis in the HK-2 cells. However, compared with the NC group, the levels of LDH released were significantly increased (Fig. 1D, middle panel), and cell viability was markedly decreased (Fig. 1D, right panel). On the basis of these results, it could be deduced that a certain concentration of $\mathrm{CaOx}$ induces the occurrence of ferroptosis, and with the occurrence of ferroptosis, the damage inflicted on HK-2 cells gradually became severe, and the degree of damage increased in a concentration-dependent manner.

CaOx-induced ferroptosis decreases the resistance of $H K-2$ cells to oxidative stress. Immunofluorescence detection of the relative fluorescence intensity of ACSL4, TF, TRC, XCT and GPX4 was then performed. Compared with the $\mathrm{CaOx}$ group, treatment with the agonist, erastin, increased the relative expression of ACSL4, TF and TRC, and reduced that of XCT and GPX4. Treatment with the inhibitor, ferrostatin-1, increased the relative expression of XCT and GPX4, and reduced that of ACSL4, TF and TRC (Figs. 2A and 3A). Western blot analysis revealed similar results as those obtained with immunofluorescence assay. However, the p53 protein level was not markedly affected by erastin and ferrostatin-1 treatment alone (Figs. 2B and C, and $3 \mathrm{~B}$ and $\mathrm{C}$ ). On the basis of the results of protein level analyses, the effectiveness of erastin and ferrostatin-1 in regulating the degree of ferroptosis occurrence in the HK-2 cells under a high-concentration $\mathrm{CaOx}$ environment was fully demonstrated. Under the condition wherein the degree of ferroptosis could be controlled in the $\mathrm{CaOx}$ environment, it was identified that the levels of intracellular CAT, T-AOC, SOD and GSH decreased with the increase in ferroptosis, and vice versa (Fig. 4A). The results obtained from the aforementioned experiments confirmed that erastin and ferrostatin-1 regulated the degree of ferroptosis in HK-2 cells in a high-concentration $\mathrm{CaOx}$ environment. Intracellular $\mathrm{Fe}^{2+}$ overload and upregulation of lipid peroxidation are necessary conditions for ferroptosis. The agonist, erastin, significantly increased the intracellular $\mathrm{Fe}^{2+}$ and MDA concentrations, while the inhibitor, ferrostatin-1, notably decreased the intracellular $\mathrm{Fe}^{2+}$ and MDA concentrations (Fig. 4B and C). These results revealed that ferroptosis induced by high concentrations of $\mathrm{CaOx}$ could reduce the effectiveness of antioxidants and antioxidant enzymes in cells, thereby affecting the ability of the cells to resist oxidative stress in response to external stimuli. 
A
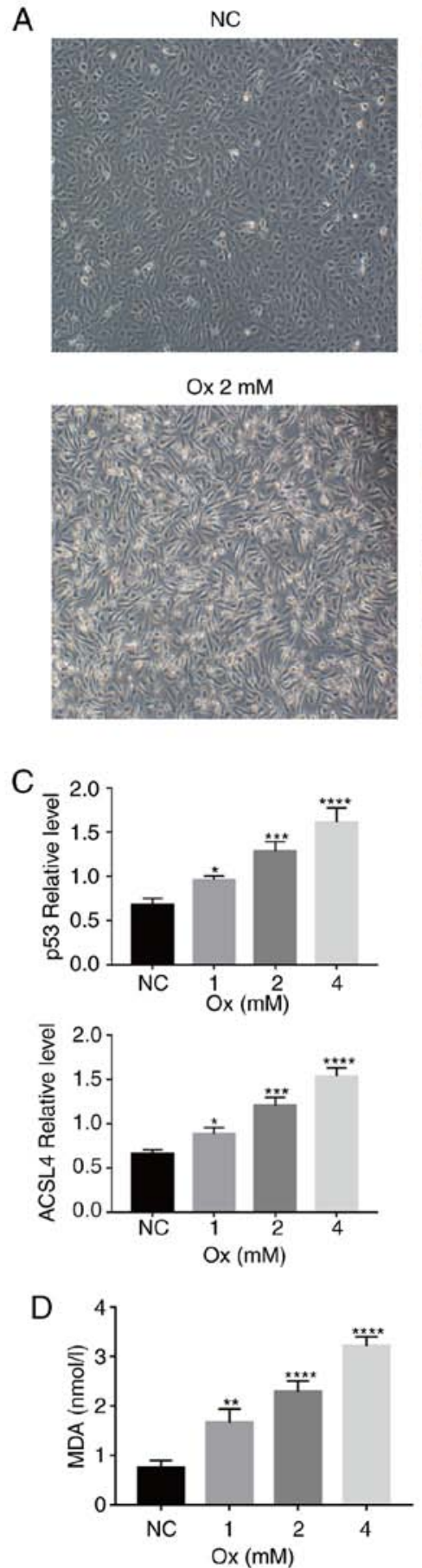
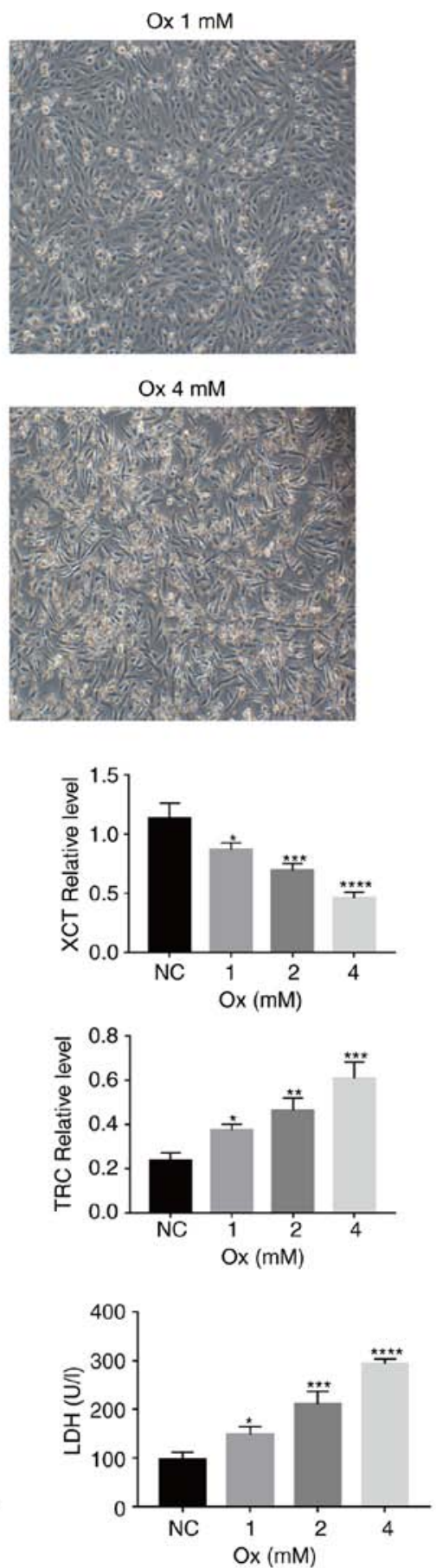

B
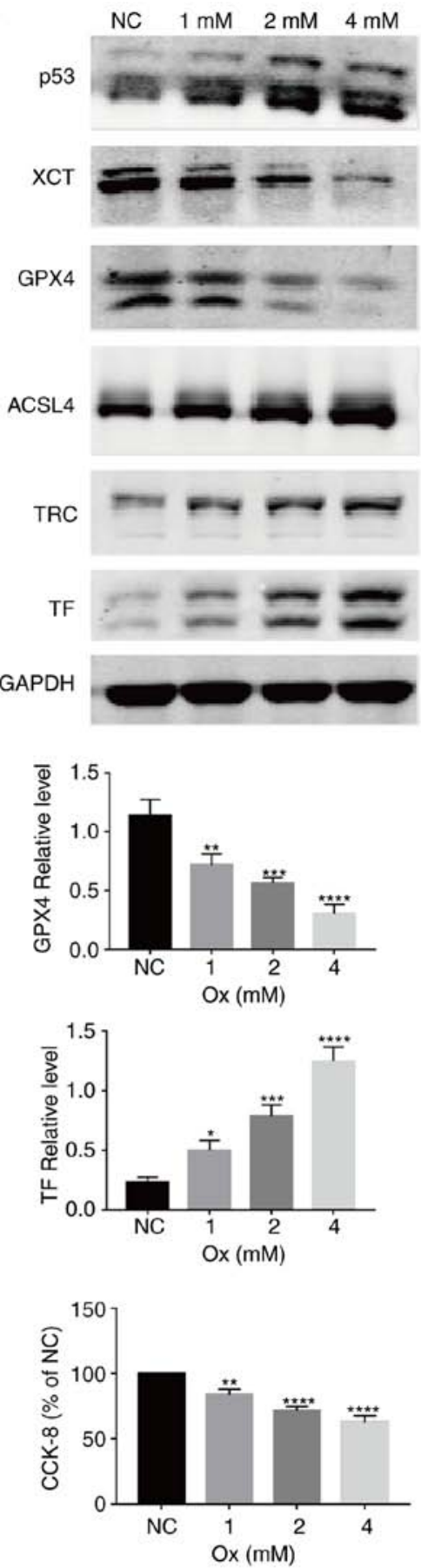

Figure 1. $\mathrm{CaOx}$ induces ferroptosis and injury to HK-2 cells in a concentration-dependent manner. HK-2 cells were treated with graded concentrations of $\mathrm{CaOx}$ for 24 h. (A) Morphological changes of HK-2 cells under various concentrations of Ox were observed by an inverted fluorescence microscope. Images were captured under bright-field (magnification, x200). One set of representative images of 3 independent experiments is shown. (B) Western blot analysis of the expression of ferroptosis-related proteins was performed. GAPDH was recruited as a loading control. (C) Representative blots and quantification analysis of ferroptosis-related proteins in HK-2 cells from the groups of graded CaOx-concentrations. Data are presented as the means \pm SEM ( $=3$ ). (D) Changes in lipid peroxidation, cell injury and the viability of HK-2 cells from the groups of graded Ox concentrations. Data are presented as the means \pm SEM ( $=3$ ). $\mathrm{CaOx} / \mathrm{Ox}$, calcium oxalate; ACSL4, long-chain acyl-CoA synthetase 4 ; TF, transferrin; TRC, transferrin receptor. ${ }^{*} \mathrm{P}<0.05,{ }^{* *} \mathrm{P}<0.01,{ }^{* * * *} \mathrm{P}<0.001,{ }^{* * * *} \mathrm{P}<0.0001$ vs. the control (NC) group.

Changes in mitochondria and ROS in HK-2 cells. The results of the above-mentioned analyses demonstrated that with the increase in the degree of ferroptosis, the overall resistance of HK-2 cells to oxidation weakened, and the degree of lipid peroxidation increased in the high-concentration $\mathrm{CaOx}$ environment. By analyzing the fluorescence ratio by JC-1 assay, it was identified that as the degree of ferroptosis increased, the mitochondrial membrane potential significantly decreased, and vice versa (Fig. 5A). Furthermore, by measuring the intensity of the MitoSox probe signal in the cell, it was demonstrated that with the increase in the degree of ferroptosis, the levels of ROS increased significantly and vice versa (Fig. 5B). From these results, it was considered that the gradual increase in ferroptosis leads to the weakening of the overall antioxidant 

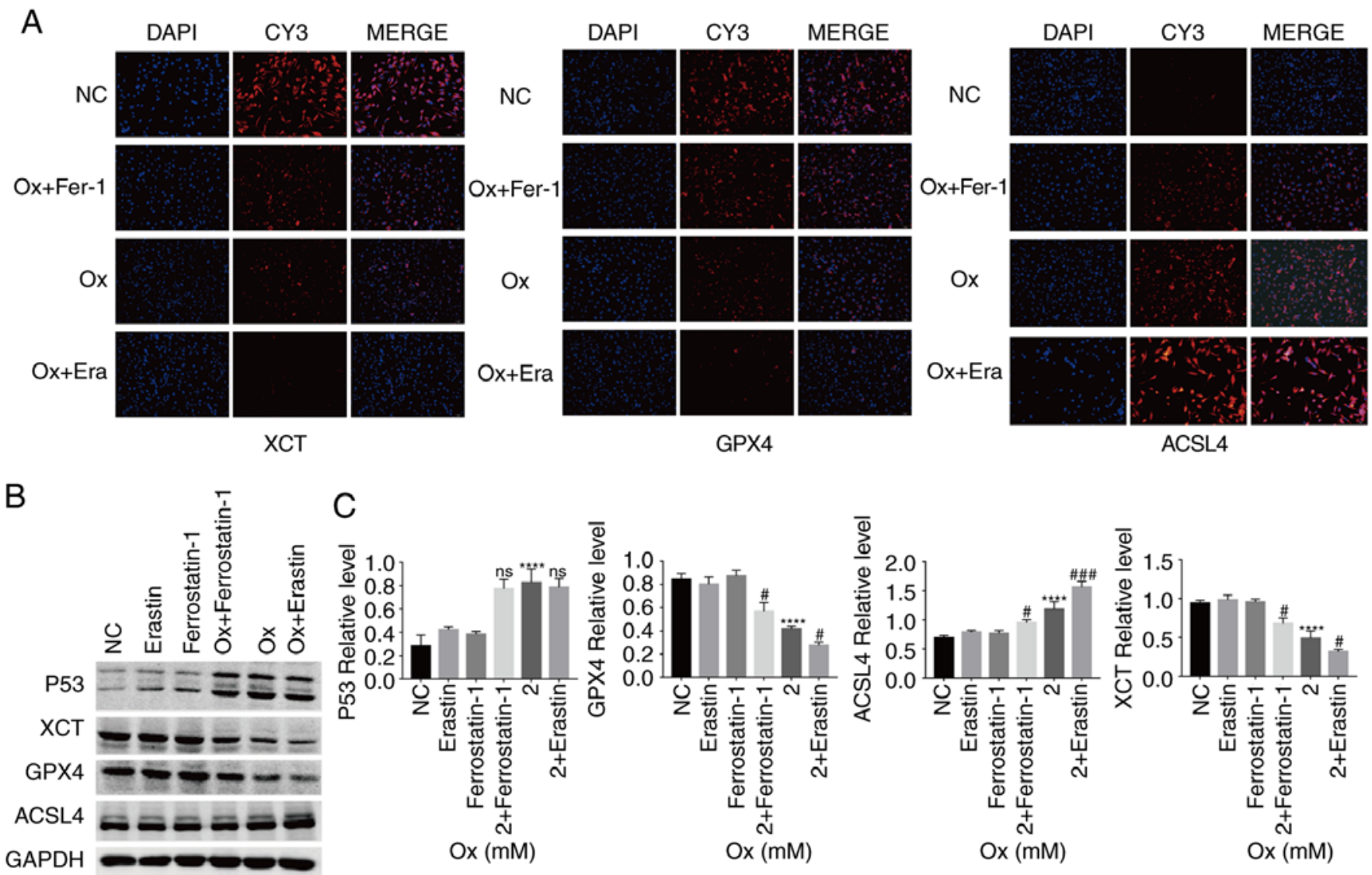

Figure 2. Quantitative analysis of the expression of ferroptosis-related lipid peroxidation signaling pathway proteins. HK-2 cells were treated with erastin $(10 \mu \mathrm{M})$ and ferrostatin-1 $(8 \mu \mathrm{M})$ with or without $\mathrm{CaOx}$ intervention solution $(2 \mathrm{mM})$ for $24 \mathrm{~h}$. (A) Immunofluorescence analysis of the expression of ferroptosis-related proteins was performed. Images were captured under a dark field (magnification, x200). One set of representative images of 3 independent experiments is shown. (B) Western blot analysis of the expression of ferroptosis-related proteins were performed. GAPDH was recruited as a loading control. (C) Representative blots and quantification analysis of ferroptosis-related proteins in HK-2 cells. Data are presented as the means \pm SEM ( $\mathrm{n}=3$ ). CaOx/Ox, calcium oxalate; Era, erastin; Fer-1, ferrostatin-1; ACSL4, long-chain acyl-CoA synthetase 4; ns, not significant. ${ }^{* * * *} \mathrm{P}<0.0001$ vs. the control (NC) group; ${ }^{\#} \mathrm{P}<0.05,{ }^{\# \# \#} \mathrm{P}<0.001$ vs. the Ox group.

capacity of cells. The mitochondria are important subcellular structures that regulate the ROS levels. The experiments of the present study demonstrated that the mitochondria were damaged with the strengthening of external stimuli, and when the mitochondria were severely damaged, they lost their ability to regulate ROS, which further led to an increase in ROS generation in cells.

Ferroptosis promotes injury to HK-2 cells under a high concentration of $\mathrm{CaOx}$. TUNEL staining was used to observe cell death in the different treatment groups. It was identified that as the degree of ferroptosis increased, the proportion of TUNEL-positive cells significantly increased, suggesting that ferroptosis promoted HK-2 cell death under a high concentration of $\mathrm{CaOx}$ (Fig. 5C). The present study also examined cell viability and LDH release levels. With the increase in the degree of ferroptosis, the LDH release levels significantly increased and cell viability significantly decreased, and vice versa (Fig. 5C). From these results, it could be inferred that $\mathrm{CaOx}$-induced ferroptosis, as observed in the previous series of biochemical reactions, reduced the ability of cells to resist oxidation, thereby resulting in $\mathrm{Fe}^{2+}$ overload, mitochondrial damage and an increase in lipid peroxidation, which then induced an intracellular ROS outbreak, eventually causing severe cell injury.
Regulating the degree of ferroptosis in a rat model. By using a rat model, the results of the in vitro experiments were confirmed in terms of ferroptosis-related proteins. Compared with the urolithiasis model group, the ferroptosis agonist, erastin, significantly increased the relative expression of ACSL4, TF and TRC, and reduced that of XCT and GPX4. The ferroptosis inhibitor, ferrostatin-1, significantly increased the relative expression of XCT and GPX4, and reduced that of ACSL4, TF and TRC (Fig. 6A). These results were similar to those obtained in the in vitro experiments.

Crystal deposition and pathological changes in renal tissue. Von Kossa staining was used to evaluate crystal deposition and pathological changes in renal tissue. Von Kossa-stained slices from the NC group exhibited a normal histological morphology and no pathological changes. Compared with the NC group, crystal formation in the urolithiasis model group was significantly increased, and renal tubules were dilated and vacuolated, leading to glomerular degeneration. The ferroptosis agonist, erastin, exacerbated the aforementioned pathological changes, while the ferroptosis inhibitor, ferrostatin-1, alleviated these pathological changes (Fig. 6B). The Von Kossa-stained slices were scored for the histological analysis of the renal tubules, and the same results were obtained as those for Von Kossa 
A

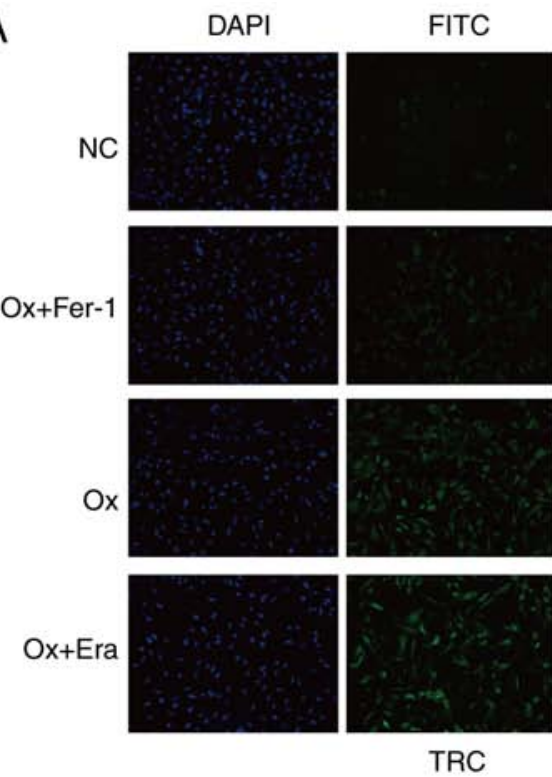

B

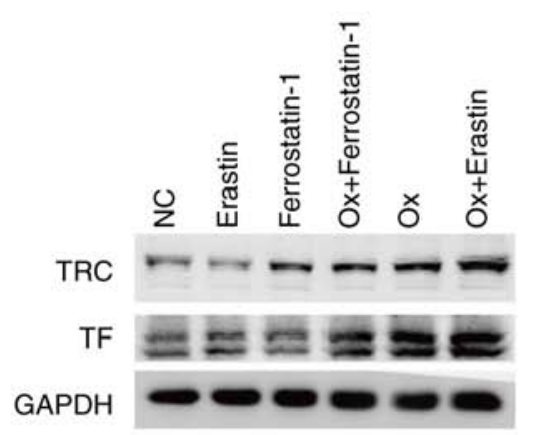

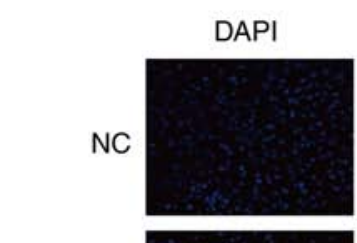
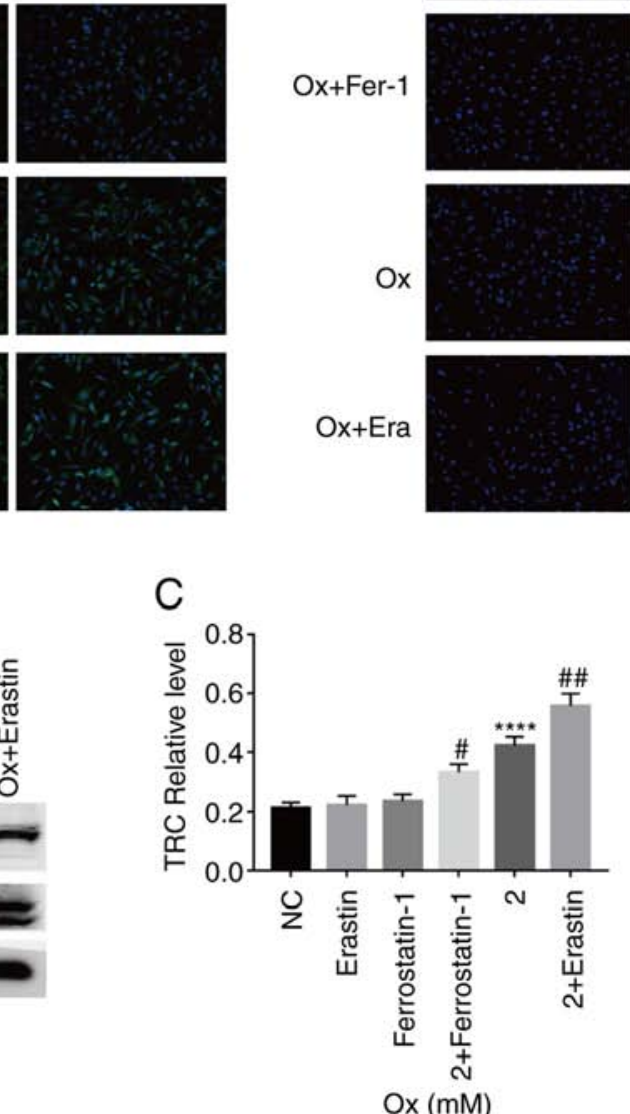

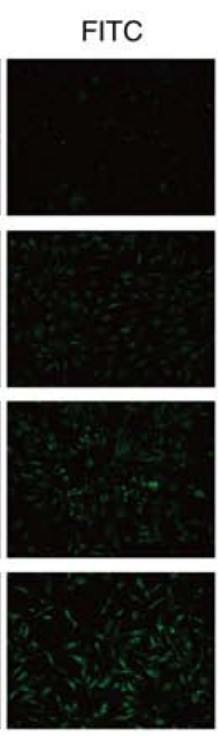

MERGE

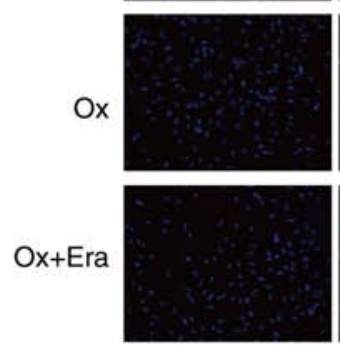

TF
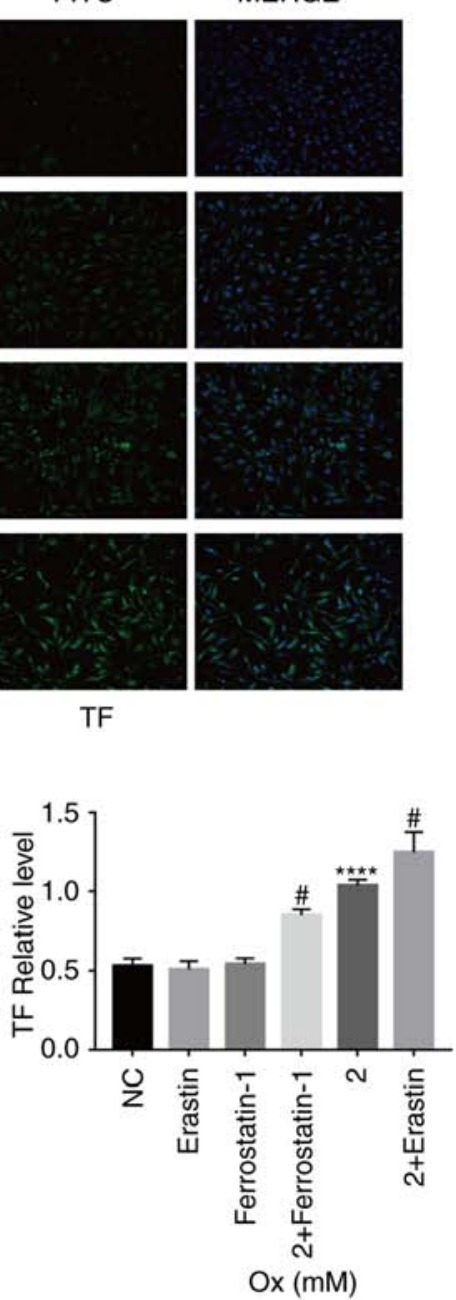

Figure 3. Quantitative analysis of the expression of ferroptosis-related $\mathrm{Fe}^{2+}$ overload signaling pathway proteins. $\mathrm{HK}-2$ cells were treated with erastin $(10 \mu \mathrm{M})$ and ferrostatin-1 $(8 \mu \mathrm{M})$ with or without CaOx intervention solution ( $2 \mathrm{mM})$ for $24 \mathrm{~h}$. (A) Immunofluorescence analysis of the expression of ferroptosis-related proteins were performed. Images were captured under a dark field (magnification, $\mathrm{x} 200$ ). One set of representative images of 3 independent experiments is shown. (B) Western blot analysis of the expression of ferroptosis-related proteins were performed. GAPDH was recruited as a loading control. (C) Representative blots and quantification analysis of ferroptosis-related proteins in HK-2 cells. Data are presented as the means \pm SEM ( $=3$ ). CaOx/Ox, calcium oxalate; Era, erastin; Fer-1, ferrostatin-1; TF, transferrin; TRC, transferrin receptor. ${ }^{* * * *} \mathrm{P}<0.0001$ vs. the control (NC) group; ${ }^{\#} \mathrm{P}<0.05$, ${ }^{\# \#} \mathrm{P}<0.01 \mathrm{vs}$. the Ox group.

staining (Fig. 6B). These results demonstrated that ferroptosis aggravated renal crystal deposition and pathological changes in the rat model of urolithiasis.

Regulating the degree of $\mathrm{Fe}^{2+}$ in renal tissue. With the increase in the degree of ferroptosis, the levels of $\mathrm{Fe}^{2+}$ in the renal tissue of rats was significantly increased, and vice versa (Fig. 6C). These results were similar to those obtained with the in vitro experiments.

Renal cell death detection. By using TUNEL staining, the effect of ferroptosis on renal cell death in the rat urolithiasis model was investigated. Compared with the NC group, the number of TUNEL-positive cells was significantly increased in the urolithiasis model group; the ferroptosis agonist, erastin, further increased the number of TUNEL-positive cells, while the ferroptosis inhibitor, ferrostatin-1, markedly reduced the number of TUNEL-positive cells (Fig. 7A). From these results, it could be suggested that ferroptosis aggravated renal cell death in the rat model of urolithiasis.
Changes in renal function in the rat model. Compared with the $\mathrm{NC}$ group, the levels of CRE and BUN in the urolithiasis model group were significantly increased. The ferroptosis agonist, erastin, reduced the clearance rate of CRE and BUN, while the ferroptosis inhibitor, ferrostatin-1, significantly improved the clearance rate of CRE and BUN (Fig. 7B). Furthermore, compared with the $\mathrm{NC}$ group, the levels of the renal tubular injury markers, NGAL and KIM-1, were significantly increased in the urolithiasis model group. The ferroptosis agonist, erastin, further increased the levels of NGAL and KIM-1, while the ferroptosis inhibitor, ferrostatin-1, significantly downregulated the levels of NGAL and KIM-1 (Fig. 7B), which was consistent with the results noted for CRE and BUN. These results indicate that ferroptosis can further damage the renal function of rats with urolithiasis. Based on the above-mentioned results, it was recognized that ferroptosis is a phenotype caused by multiple signaling pathways and multiple factors. The level of antioxidants in the microenvironment and the expression of ferroptosis-related proteins all have a marked impact on the degree of ferroptosis (Fig. 8). 

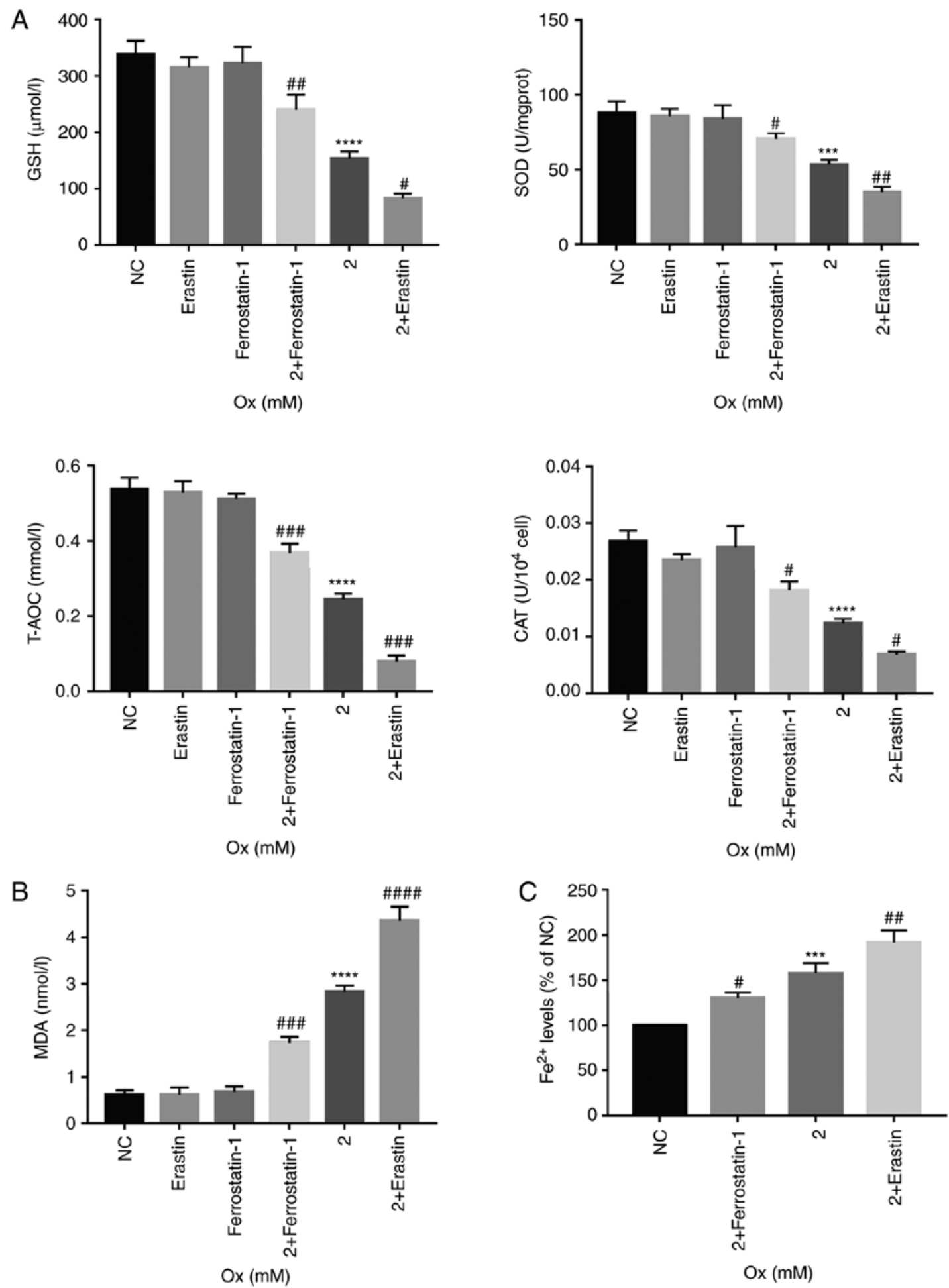

Figure 4. Changes in antioxidant capacity, lipid peroxidation and concentration of $\mathrm{Fe}^{2+}$ in $\mathrm{HK}-2$ cells induced by ferroptosis. HK-2 cells were treated with erastin $(10 \mu \mathrm{M})$ and ferrostatin-1 $(8 \mu \mathrm{M})$ with or without $\mathrm{CaOx}$ intervention solution $(2 \mathrm{mM})$ for $24 \mathrm{~h}$. (A) Quantification analysis of antioxidant markers in HK-2 cells. Data are presented as the means \pm SEM $(n=3)$. (B) Quantification analysis of lipid peroxidation levels in HK-2 cells. Data are presented as the means \pm SEM $(n=3)$. (C) Quantification analysis of Fe2+ levels in HK-2 cells. Data are presented as the means \pm SEM $(n=3)$. CaOx/Ox, calcium oxalate; GSH, glutathione; SOD, superoxide dismutase; T-AOC, total antioxidant capacity; CAT, catalase; MDA, malondialdehyde. ${ }^{* * * *} \mathrm{P}<0.001,{ }^{* * * * *} \mathrm{P}<0.0001$ vs. the control (NC) group; ${ }^{\#} \mathrm{P}<0.05,{ }^{\# \#} \mathrm{P}<0.01,{ }^{\# \#} \mathrm{P}<0.001,{ }^{\# \# \#} \mathrm{P}<0.0001$ vs. the Ox group.

\section{Discussion}

Ferroptosis, as a regulated cell death phenomenon, was first reported in 2012, and is a type of iron-dependent lipid peroxidation (5). With the advancements in research, ferroptosis has been reported to play a role in the development of blood diseases, neurological diseases, respiratory diseases, cardiovascular diseases, reproductive system diseases and tumors (28-32). However, the related reports of the role of ferroptosis in urinary system diseases have mainly focused 
A

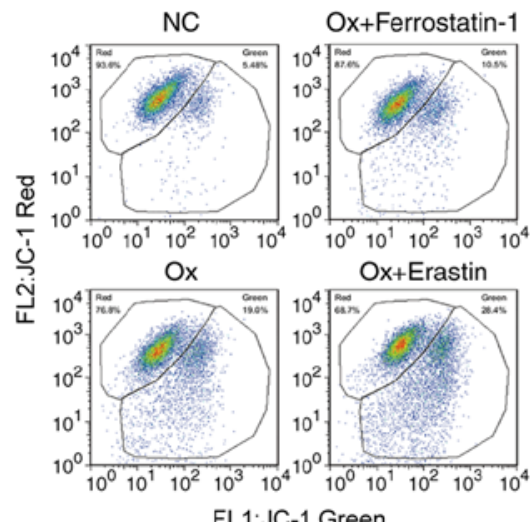

FL1:JC-1 Green

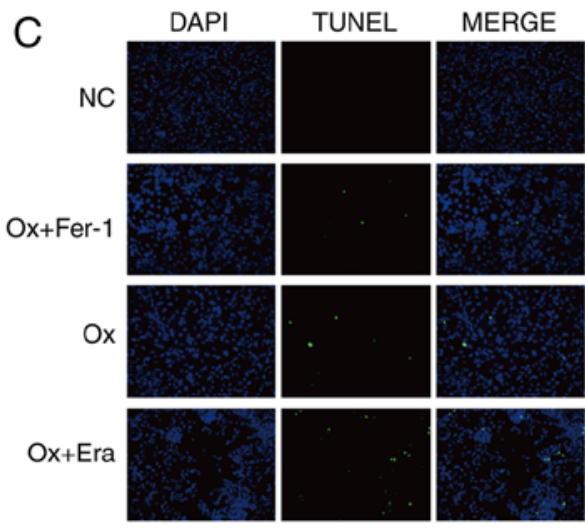

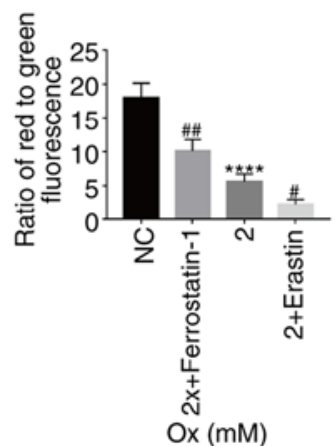

$B$

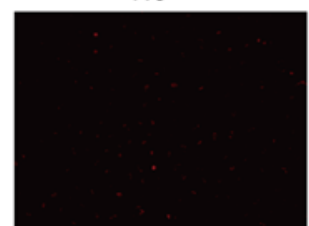

$\mathrm{Ox}$

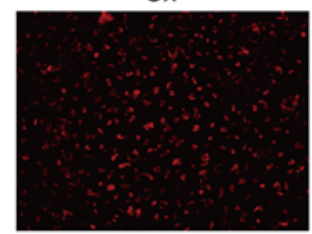

Ox+Ferrostatin-1

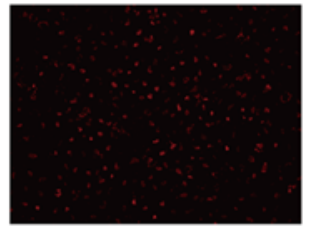

Ox+Erastin

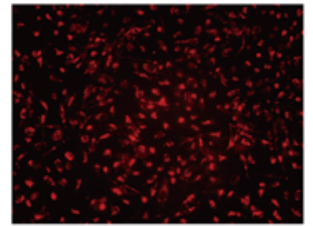

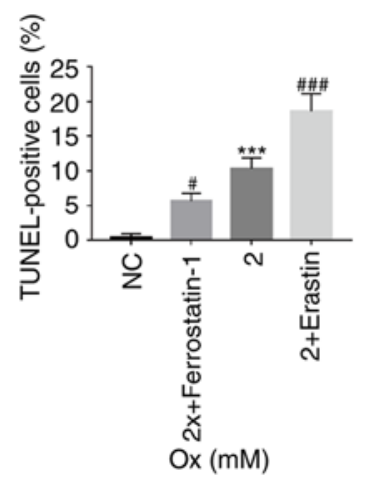
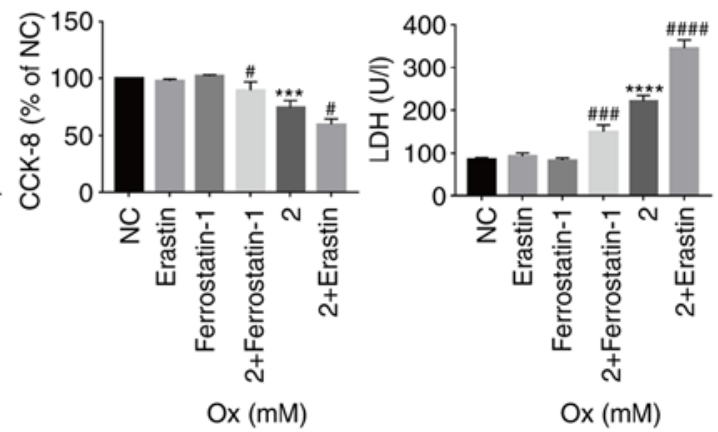

Figure 5. Effect of the degree of ferroptosis on injury to HK-2 cells exposed to a high concentration of CaOx. HK-2 cells were treated with erastin (10 $\mu \mathrm{M})$ and ferrostatin-1 $(8 \mu \mathrm{M})$ with or without CaOx intervention solution $(2 \mathrm{mM})$ for $24 \mathrm{~h}$. (A) JC-1 analysis of mitochondrial membrane potential in HK-2 cells. Data are presented as the means \pm SEM ( $n=3$ ). (B) MitoSox analysis of ROS levels in HK-2 cells. Images were captured under a dark field (magnification, $x 200)$. One set of representative images of 3 independent experiments is shown. (C) TUNEL analysis of cell death in HK-2 cells. Images were captured under a dark field (magnification, $\mathrm{x} 200$ ). One set of representative images of 3 independent experiments is shown. Data are presented as the means $\pm \mathrm{SEM}$ ( $\mathrm{n}=3$ ). CCK-8 and LDH analysis of cell death in HK-2 cells. Data are presented as the means \pm SEM ( $n=3)$. CaOx/Ox, calcium oxalate; Era, erastin; Fer-1, ferrostatin-1. ${ }^{* * *} \mathrm{P}<0.001$,

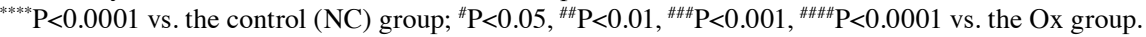

on renal failure and renal tumors $(18,22)$, and to date, there is no relevant report available on the topic of urolithiasis, at least to the best of our knowledge. Through in vivo and in vitro experiments, the present study, for the first time, to the best of our knowledge, provided strong evidence of the important role of ferroptosis in the formation of urolithiasis induced by high concentrations of $\mathrm{CaOx}$. The results of the in vitro experiments demonstrated that as the concentration of $\mathrm{CaOx}$ increased, the viability of the $\mathrm{HK}-2$ cells gradually decreased, the cell morphology gradually changed from an oval to a long spindle-like shape, and the levels of LDH release and lipid peroxidation increased significantly. Moreover, with the increase in the $\mathrm{CaOx}$ concentration, the relative expression levels of ferroptosis-related signaling pathway proteins (p53, ACSL4, TF, and TRC) significantly increased, while the relative expression levels of XCT and GPX4 decreased. Thus, it was observed that cell injury and the degree of ferroptosis exhibits a positive association with the increase in the $\mathrm{CaOx}$ concentration. A high concentration of $\mathrm{CaOx}$ led to markedly increased ferroptosis and injury in the HK-2 cells. With the accumulation of $\mathrm{Fe}^{2+}$ and the increase in lipid peroxidation, the mitochondria were damaged, leading to the gradual weakening of the overall antioxidant capacity of cells and the rapid release of ROS, which eventually resulted in oxidative stress injury $(33,34)$. Therefore, the present study wished to determine the mutual association between ferroptosis and high-concentration $\mathrm{CaOx}$-induced urolithiasis from the aspects of $\mathrm{Fe}^{2+}$ levels, cell antioxidant capacity, lipid peroxidation levels, mitochondrial damage, ROS accumulation levels and cell death.

To further investigate the association between ferroptosis and injury to HK-2 cells induced by exposure to high concentrations of $\mathrm{CaOx}$, the classic agonist, erastin, and the ferroptosis inhibitor, ferrostatin-1, were used (33). It was identified that erastin significantly increased the relative expression of the ferroptosis-promoting signaling pathway proteins, ACSL4, TF and TRC, and reduced the relative expression of the ferroptosis-inhibiting signaling pathway proteins, XCT and GPX4. As was expected, ferrostatin-1 induced the exact opposite effect. It was also identified that the relative expression of was is not affected by erastin and ferrostatin-1. Thus, it was demonstrated that the regulation of ferroptosis by erastin and ferrostatin-1 began with the cystine/glutamate transport system. As regards cell biochemical markers, in the urolithiasis model, erastin significantly reduced the antioxidant capacity of HK-2 cells, leading to an increase in the levels of mitochondrial membrane potential, lipid peroxidation and ROS. At the same time, the concentration of intracellular $\mathrm{Fe}^{2+}$ markedly increased, as well as the degree of cell damage, and vice versa (23-27,33-35). From the aforementioned evidence, it 

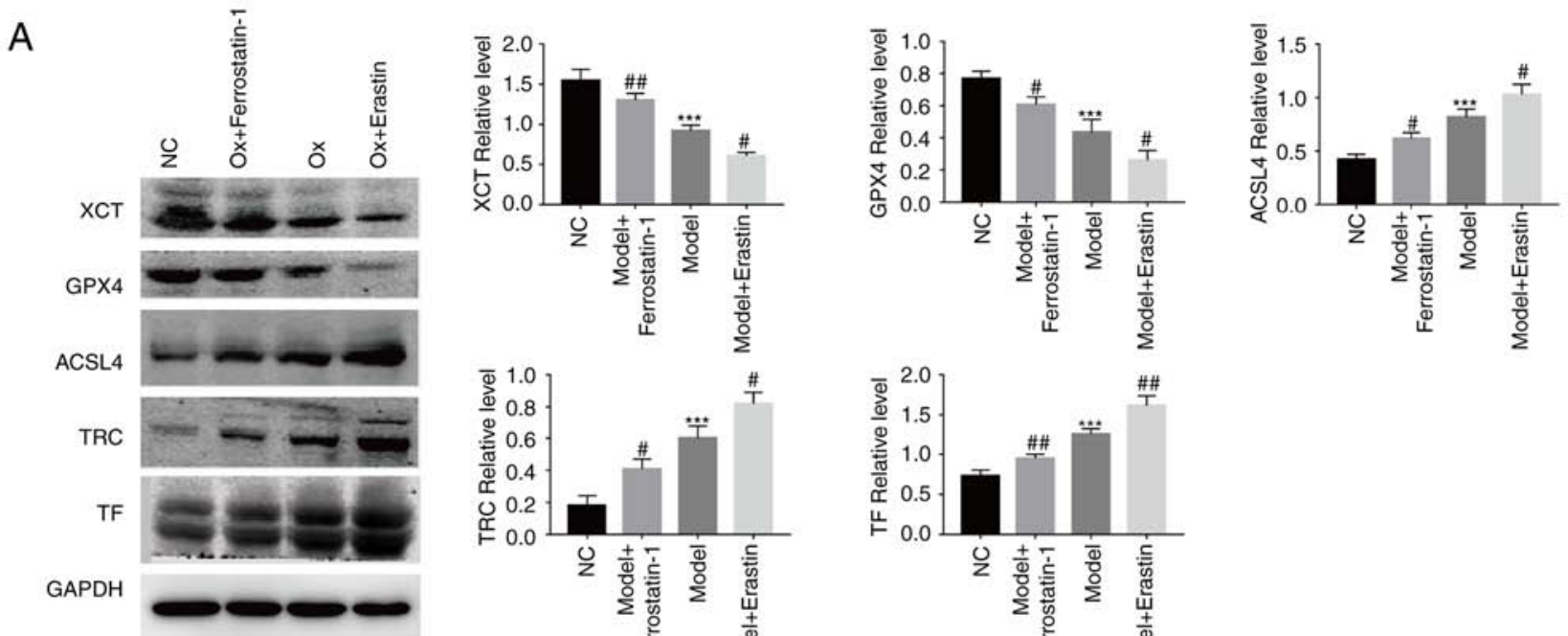

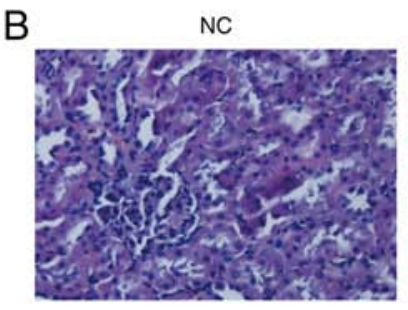

Ox

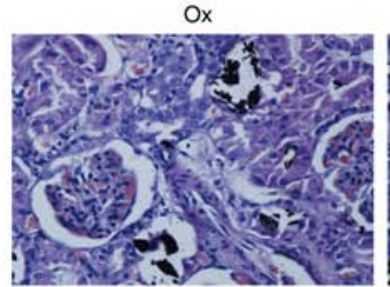

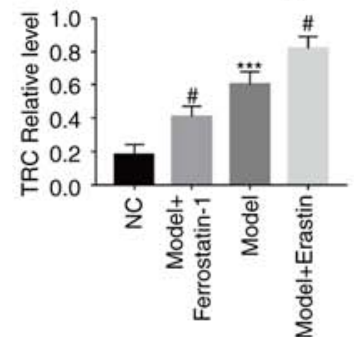

Ox+Ferrostatin-1

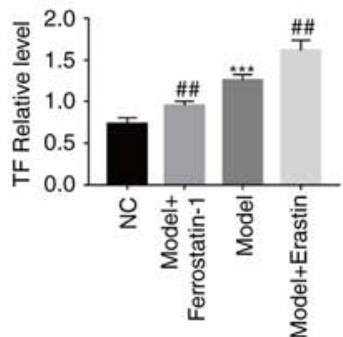

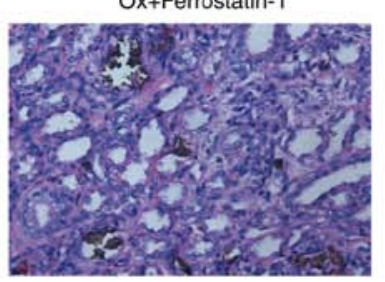

Ox+Erastin

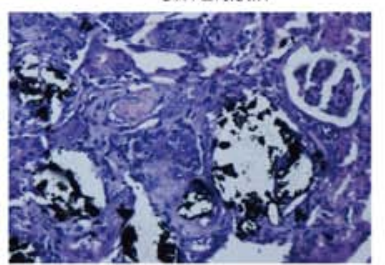

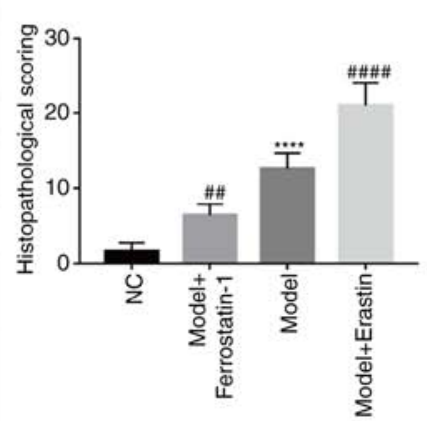

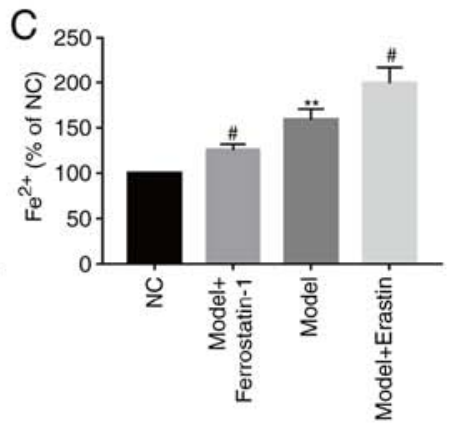

Figure 6. Ferroptosis and renal tissue injury in a rat model. Rat models of nephrolithiasis were established by the administration of $0.75 \%$ ethylene glycol (EG) in the drinking water for 4 weeks. The rats were randomly divided into 4 groups of 8: The NC group, nephrolithiasis model group, nephrolithiasis model treatment with Erastin (10 mg/kg/day) group, and nephrolithiasis model treatment with Ferrostatin-1 (4 mg/kg/day) group. (A) Western blot analysis of the expression of ferroptosis-related proteins were performed. GAPDH was recruited as a loading control. Representative blots and quantification analysis of ferroptosis-related proteins in rat renal tissues. Data are presented as the means \pm SEM ( $=3$ ). (B) Histopathological analysis of Von Kossa staining slices was performed. One set of representative images of 5 independent experiments is shown. Data are presented as the means \pm SEM ( $\mathrm{n}=5$ ). $(\mathrm{C}) \mathrm{Quantification}$ analysis of $\mathrm{Fe}^{2+}$ levels in rat renal tissues. Data are presented as the means $\pm \mathrm{SEM}(\mathrm{n}=3) .{ }^{* *} \mathrm{P}<0.01,{ }^{* * *} \mathrm{P}<0.001,{ }^{* * * *} \mathrm{P}<0.0001$ vs. the control $(\mathrm{NC})$ group; ${ }^{\#} \mathrm{P}<0.05,{ }^{\# \#} \mathrm{P}<0.01$,

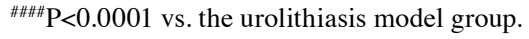

was concluded that a high concentration of $\mathrm{CaOx}$ could induce ferroptosis, and the increase in ferroptosis levels aggravated lipid peroxidation levels and mitochondrial injury, leading to an overload of ROS in cells and eventually increasing the degree of cell injury. By reducing the degree of ferroptosis, the damage and death of HK-2 cells caused by the aforementioned effects could be reduced. Studies have demonstrated that a high $\mathrm{CaOx}$ concentration in urine and crystal deposition could induce the production of ROS and damage renal epithelial cells $(36,37)$, and that this damage to renal tubular epithelial cells plays a vital role in the formation of $\mathrm{CaOx}$ kidney stones (38). From the aforementioned results and the findings of the in vitro experiments, it was concluded that ferroptosis promoted the formation of urolithiasis.

In the rat model of urolithiasis, the same result as that obtained in the in vitro experiment was observed. By performing Von Kossa staining, it was identified that with the increase in the degree of ferroptosis, the deposition of crystals in the kidneys significantly increased, and the pathological fraction of renal tubules markedly increased. Serum CRE and BUN are classic clinical diagnostic markers for investigating renal function, while NGAL and KIM-1 are more widely used as clinical diagnostic indicators of early renal function (39). The present study found that with the increase in the degree of ferroptosis, renal function in the rat model of urolithiasis was significantly reduced, and the number of TUNEL-positive cells in renal tissues was notably increased. This phenomenon was suppressed by ferroptosis inhibitor. These data once again confirmed the promoting effect of ferroptosis in the formation of urolithiasis

Through a series of in vivo and in vitro experiments, the present study inferred that when exposed to a high concentration of $\mathrm{CaOx}$, crystal deposition occurred as $\mathrm{CaOx}$ was in a supersaturated state, leading to the occurrence of cell-crystal reactions, which inhibited the transcriptional regulation of the cystine/glutamate transport system. Furthermore, the intracellular transport of cystine was affected, leading to the inhibition of intracellular GSH synthesis and downregulation 
A
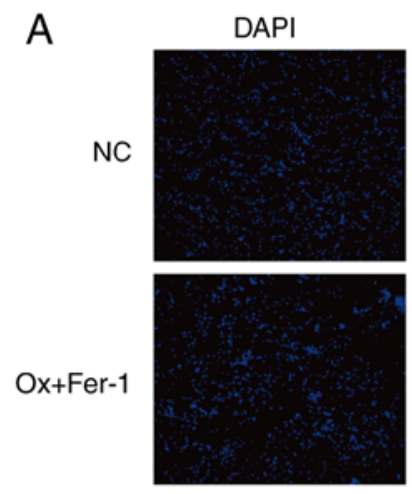

$\mathrm{Ox}$
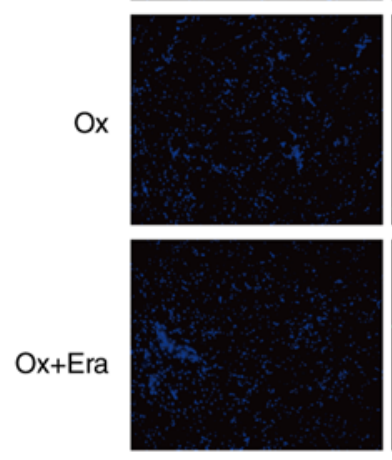

TUNEL

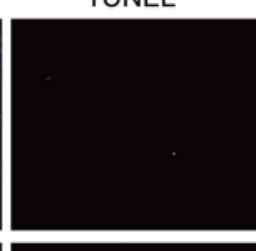

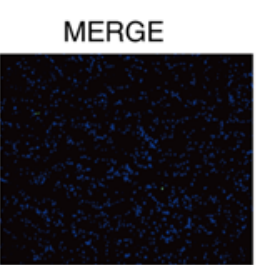
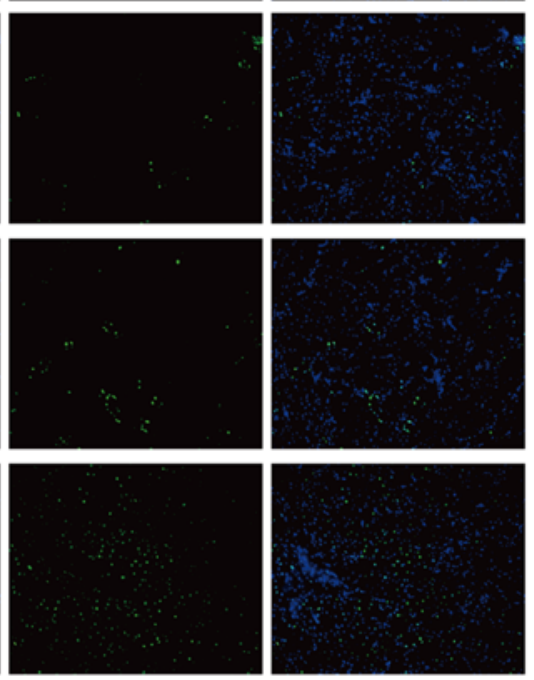
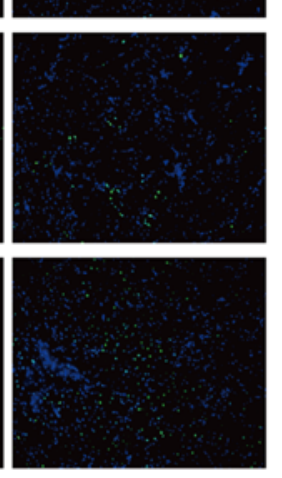
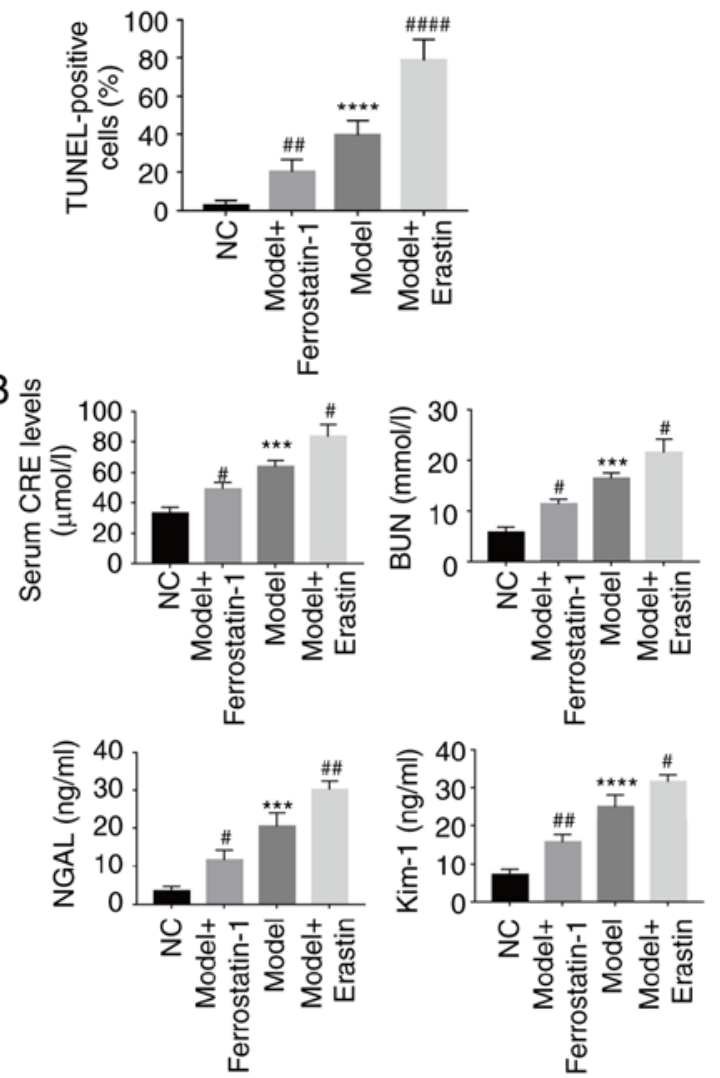

Figure 7. Renal tissue and functional injury induced by CaOx-induced ferroptosis. (A) TUNEL analysis of renal tissues injury in rat model. Images were captured under dark-field (magnification, $x 200$ ). One set of representative images of 3 independent experiments is shown. Data are presented as the means \pm SEM ( $=3$ ). (B) Quantitative analysis of renal function and renal injury markers. Data are presented as the means $\pm \mathrm{SEM}$ ( $\mathrm{n}=3$ ). CaOx/Ox, calcium oxalate; Era, erastin; Fer-1, ferrostatin-1. ${ }^{* * *} \mathrm{P}<0.001,{ }^{* * * *} \mathrm{P}<0.0001$ vs. the control group; ${ }^{\#} \mathrm{P}<0.05,{ }^{\# \#} \mathrm{P}<0.01,{ }^{\# \# \# \#} \mathrm{P}<0.0001$ vs. the Ox group or the urolithiasis model group.

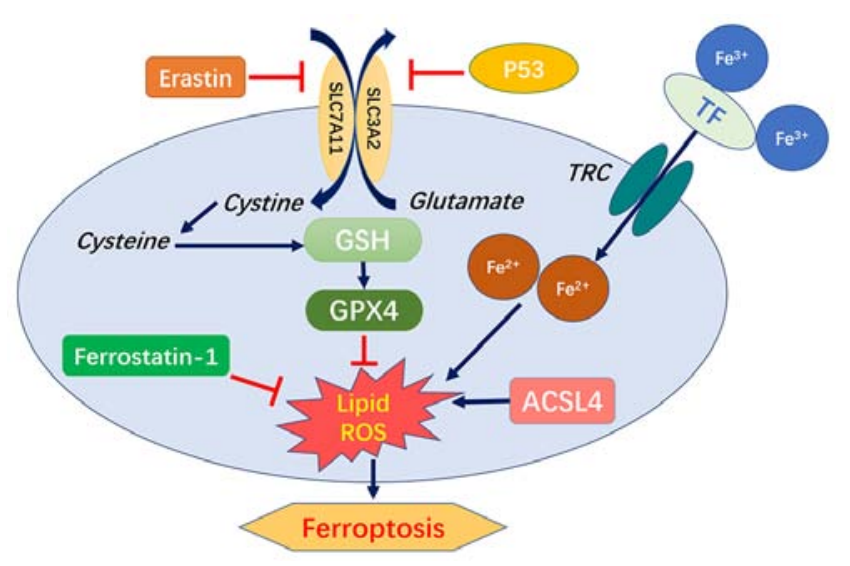

Figure 8. Signaling pathway of $\mathrm{CaOx}$-induced ferroptosis in $\mathrm{HK}-2$ cells and rat renal tissues. SLC7A11/SLC3A2, cystine/glutamate transport system; TF, transferrin; TRC, transferrin receptor; GPX4, glutathione peroxidase 4; ACSL4, Acyl-CoA synthetase long-chain family 4.

of intracellular activity of GPX4, eventually resulting in lipid peroxidation. On the other hand, the cell-crystal response activated the function of the TRC-TF complex, causing a large amount of iron ions to be transported into cells, and then through the redox reaction, resulting in an excess of intracellular $\mathrm{Fe}^{2+}$. The combined effects of these factors caused lipid peroxidation and mitochondrial injury, and then triggered the burst of ROS, resulting in cell injury, which eventually produced initial conditions for the formation of urolithiasis. In the ferroptosis-lipid peroxidation-ROS injury mode, the damage to renal tubular epithelial cells induced a more severe state of crystal deposition, which triggered a positive feedback cell-crystal injury reaction, and eventually led to the formation of kidney stones. Therefore, the formation of ferroptosis-induced urolithiasis is a complex injury mode.

The present study demonstrated that ferroptosis plays an important role in the formation of urolithiasis; however, the formation of urolithiasis is a complex pathological process, and ferroptosis does not play the only role in the pathological process alone. In some previous studies, it was reported that a high concentration of $\mathrm{CaOx}$ crystals induced an excessive burst of autophagy and ERS (14-16), which led to the subsequent oxidative stress reaction and eventually induced the formation of kidney stones. As an important component of urolithiasis, whether ferroptosis has a crosstalk with autophagy and ERS, and whether it affects the formation of urolithiasis through a synergistic effect, has not been reported in the field of urolithiasis, at least to the best of our knowledge. However, in other related studies, a close interaction between ferroptosis, autophagy and ERS has been shown $(18,20)$. Some researchers have considered that autophagy was a form of autophagy cell death (12). Mechanistically, autophagy promotes ferroptosis via a form of cargo-specific autophagy known as ferrotinophagy. The upregulation of autophagy levels can cause the overexpression of the NCOA4 gene, and NCOA4 can cause iron storage 
as a cargo receptor. The autophagy degradation of protein ferritin induces the overload of intracellular $\mathrm{Fe}^{2+}$ and causes ferroptosis (40). On the other hand, it has been demonstrated that beclin-1 can not only affect the regulation of autophagy as an autophagy regulator, but also affect the intake of cystine by combining with XCT to form the beclin-1/XCT complex, which leads to the inhibition of GPx4 synthesis and the increase of lipid peroxidation level (41). Finally, the Beclin1-XCT signaling pathway can promote ferroptosis. At the same time, ATF4 is a critical mediator of metabolic and oxidative homeostasis and cell survival; as a key regulator of the p-ERK signaling pathway in the ERS process, it plays a positive regulatory role on ERS. On the other hand, ATF4 can inhibit the cystine transport function of XCT via the ATF4/CHOP/CHAC1 signaling pathway, which will further hinder the synthesis of GSH and decrease the expression of GPX4, which eventually leads to ferroptosis (42). Therefore, there is a complex crosstalk between ferroptosis, autophagy and ERS, which can be regulated by multiple signaling pathways, and there is a common target among multiple signaling pathways, which can achieve multi-level regulation by regulating a common target. This makes it more practical to study the interaction mechanism of ferroptosis, autophagy and ERS in the formation of kidney stones. On the basis of the present study, this is also the research direction of our group. Based on the experimental results and discussions presented in the current study, we have reason to consider that ferroptosis plays an important role in the formation of urolithiasis, and it has been reported that ferroptosis is different from irreversible injury caused by apoptosis. Ferroptosis is reversible (43), which has made research on ferroptosis and its relation to the formation of urolithiasis to have a very promising future, thus bringing a new direction for the treatment of urolithiasis.

\section{Acknowledgements}

Not applicable.

\section{Funding}

The present study was supported by the National Natural Science Funds of China (grant no. 31600785 and 82070723), the Fundamental Research Funds for the Central Universities of China (grant no. 2042016kf0097) and the Applied Basic Research Programs of Wuhan Science and Technology Bureau (grant no. 2017060201010203).

\section{Availability of data and materials}

All data generated or analyzed during this study are included in this published article.

\section{Authors' contributions}

ZH designed the study. ZH, BL and QS performed the experiments and drafted the manuscript. BL, JL and YX participated in data analysis. WL, CS and SY were involved in the discussion and interpretation of the results. All authors read and approved the final manuscript.

\section{Ethics approval and consent to participate}

All animal experiment protocols were approved by the Animal Care Committee of Wuhan University (Wuhan, China) and the Laboratory Animal Welfare and Ethics Committee of Renmin Hospital of Wuhan University.

\section{Patient consent for publication}

Not applicable.

\section{Competing interests}

The authors declare that they have no competing interests.

\section{References}

1. Sorokin I, Mamoulakis C, Miyazawa K, Rodgers A, Talati J and Lotan Y: Epidemiology of stone disease across the world. World J Urol 35: 1301-1320, 2017.

2. Scales CD Jr, Tasian GE, Schwaderer AL, Goldfarb DS, Star RA and Kirkali Z: Urinary stone disease: Advancing knowledge, patient care, and population health. Clin J Am Soc Nephrol 11: 1305-1312, 2016.

3. Tsujihata M: Mechanism of calcium oxalate renal stone formation and renal tubular cell injury. Int J Urol 15: 115-120, 2008.

4. Amato M, Lusini ML and Nelli F: Epidemiology of nephrolithiasis today. Urol Int 72 (Suppl 1): S1-S5, 2004.

5. Dixon SJ, Lemberg KM, Lamprecht MR, Skouta R, Zaitsev EM, Gleason CE, Patel DN, Bauer AJ, Cantley AM, Yang WS, et al: Ferroptosis: An iron-dependent form of nonapoptotic cell death. Cell 149: 1060-1072, 2012.

6. Xie Y, Zhu S, Song X, Sun X, Fan Y, Liu J, Zhong M, Yuan H, Zhang L, Billiar TR, et al: The tumor suppressor p53 limits ferroptosis by blocking DPP4 activity. Cell Rep 20: 1692-1704, 2017.

7. Koppula P, Zhang Y, Zhuang L and Gan B: Amino acid transporter SLC7A11/xCT at the crossroads of regulating redox homeostasis and nutrient dependency of cancer. Cancer Commun (Lond) 38: 12, 2018.

8. Forcina GC and Dixon SJ: GPX4 at the crossroads of lipid homeostasis and ferroptosis. Proteomics 19: e1800311, 2019.

9. Homma T, Kobayashi S, Sato H and Fujii J: Edaravone, a free radical scavenger, protects against ferroptotic cell death in vitro. Exp Cell Res 384: 111592, 2019.

10. Li Y, Feng D, Wang Z, Zhao Y, Sun R, Tian D, Liu D, Zhang F, Ning S, Yao J and Tian X: Ischemia-induced ACSL4 activation contributes to ferroptosis-mediated tissue injury in intestinal ischemia/reperfusion. Cell Death Differ 26: 2284-2299, 2019.

11. Kagan VE, Mao G, Qu F, Angeli JP, Doll S, Croix CS, Dar HH, Liu B, Tyurin VA, Ritov VB, et al: Oxidized arachidonic and adrenic PEs navigate cells to ferroptosis. Nat Chem Biol 13: 81-90, 2017.

12. Gao M, Monian P,Pan Q,Zhang W, Xiang J and Jiang X: Ferroptosis is an autophagic cell death process. Cell Res 26: 1021-1032, 2016.

13. Ohgami RS, Campagna DR, McDonald A and Fleming MD: The steap proteins are metalloreductases. Blood 108: 1388-1394, 2006.

14. Liu Y, Li D, He Z, Liu Q, Wu J, Guan X, Tao Z and Deng Y: Inhibition of autophagy-attenuated calcium oxalate crystal-induced renal tubular epithelial cell injury in vivo and in vitro. Oncotarget 9: 4571-4582, 2017.

15. Liu Y, Liu Q, Wang X, He Z, Li D, Guan X, Tao Z and Deng Y: Inhibition of autophagy attenuated ethylene glycol induced crystals deposition and renal injury in a rat model of nephrolithiasis. Kidney Blood Press Res 43: 246-255, 2018.

16. Kang J, Sun Y, Deng Y, Liu Q, Li D, Liu Y, Guan X, Tao Z and Wang X: Autophagy-endoplasmic reticulum stress inhibition mechanism of superoxide dismutase in the formation of calcium oxalate kidney stones. Biomed Pharmacother 121: 109649, 2020.

17. Wang XF, Zhang BH, Lu XQ and Wang RQ: Gastrin-releasing peptide receptor gene silencing inhibits the development of the epithelial-mesenchymal transition and formation of a calcium oxalate crystal in renal tubular epithelial cells in mice with kidney stones via the PI3K/Akt signaling pathway. J Cell Physiol 234: 1567-1577, 2019. 
18. Kong Z, Liu R and Cheng Y: Artesunate alleviates liver fibrosis by regulating ferroptosis signaling pathway. Biomed Pharmacother 109: 2043-2053, 2019.

19. Gao M, Deng J, Liu F, Fan A, Wang Y, Wu H, Ding D, Kong D, Wang Z, Peer D and Zhao Y: Triggered ferroptotic polymer micelles for reversing multidrug resistance to chemotherapy. Biomaterials 223: 119486, 2019.

20. Park EJ, Park YJ, Lee SJ, Lee K and Yoon C: Whole cigarette smoke condensates induce ferroptosis in human bronchial epithelial cells. Toxicol Lett 303: 55-66, 2019.

21. Khan SR: Reactive oxygen species as the molecular modulators of calcium oxalate kidney stone formation: Evidence from clinical and experimental investigations. J Urol 189: 803-811, 2013.

22. Miess H, Dankworth B, Gouw AM, Rosenfeldt M, Schmitz W, Jiang M, Saunders B, Howell M, Downward J, Felsher DW, et al: The glutathione redox system is essential to prevent ferroptosis caused by impaired lipid metabolism in clear cell renal cell carcinoma. Oncogene 37: 5435-5450, 2018

23. Jurisic V, Radenkovic $S$ and Konjevic G: The actual role of LDH as tumor marker, biochemical and clinical aspects. Adv Exp Med Biol 867: 115-124, 2015.

24. Krych-Madej J and Gebicka L: Interactions of nitrite with catalase: Enzyme activity and reaction kinetics studies. J Inorg Biochem 171: 10-17, 2017

25. Owen JB and Butterfield DA: Measurement of oxidized/reduced glutathione ratio. Methods Mol Biol 648: 269-277, 2010.

26. Carillon J, Rouanet JM, Cristol JP and Brion R: Superoxide dismutase administration, a potential therapy against oxidative stress related diseases: Several routes of supplementation and proposal of an original mechanism of action. Pharm Res 30 2718-2728, 2013.

27. Del Rio D, Stewart AJ and Pellegrini N: A review of recent studies on malondialdehyde as toxic molecule and biological marker of oxidative stress. Nutr Metab Cardiovasc Dis 15: 316-328, 2005.

28. Wang F, Lv H, Zhao B, Zhou L, Wang S, Luo J, Liu J and Shang P. Iron and leukemia: New insights for future treatments. J Exp Clin Cancer Res 38: 406, 2019.

29. Masaldan S, Belaidi AA, Ayton S and Bush AI: Cellular senescence and iron dyshomeostasis in Alzheimer's disease. Pharmaceuticals (Basel) 12: E93, 2019.

30. Alvarez SW, Sviderskiy VO, Terzi EM, Papagiannakopoulos T, Moreira AL, Adams S, Sabatini DM, Birsoy K and Possemato R NFS1 undergoes positive selection in lung tumours and protects cells from ferroptosis. Nature 551: 639-643, 2017.
31. Stamenkovic A, Pierce GN and Ravandi A: Phospholipid oxidation products in ferroptotic myocardial cell death. Am J Physiol Heart Circ Physiol 317: H156-H163, 2019.

32. Ng SW, Norwitz SG and Norwitz ER: The impact of iron overload and ferroptosis on reproductive disorders in humans: Implications for preeclampsia. Int J Mol Sci 20: 3283, 2019.

33. Yang WS and Stockwell BR: Ferroptosis: Death by lipid peroxidation. Trends Cell Biol 26: 165-176, 2016.

34. Zorov DB, Juhaszova M and Sollott SJ: Mitochondrial reactive oxygen species (ROS) and ROS-induced ROS release. Physiol Rev 94: 909-950, 2014.

35. Thévenod F: Iron and its role in cancer defense: A double-edged sword. Met Ions Life Sci: doi: 10.1515/9783110470734-021.

36. Li CY, Deng YL and Sun BH: Taurine protected kidney from oxidative injury through mitochondrial-linked pathway in a rat model of nephrolithiasis. Urol Res 37: 211-220, 2009.

37. Li CY, Deng YL and Sun BH: Effects of apocynin and losartan treatment on renal oxidative stress in a rat model of calcium oxalate nephrolithiasis. Int Urol Nephrol 41: 823-833, 2009.

38. Ouyang JM, Yao XQ, Tan J and Wang FX: Renal epithelial cell injury and its promoting role in formation of calcium oxalate monohydrate. J Biol Inorg Chem 16: 405-416, 2011.

39. Kandur Y, Gonen S, Fidan K and Soylemezoglu O: Evaluation of urinary KIM-1, NGAL, and IL-18 levels in determining early renal injury in pediatric cases with hypercalciuria and/or renal calculi. Clin Nephrol 86: 62-69, 2016.

40. Hou W, Xie Y, Song X, Sun X, Lotze MT, Zeh HJ III, Kang R and Tang D: Autophagy promotes ferroptosis by degradation of ferritin. Autophagy 12: 1425-1428, 2016

41. Kang R, Zhu S, Zeh HJ, Klionsky DJ and Tang D: BECN1 is a new driver of ferroptosis. Autophagy 14: 2173-2175, 2018.

42. Wang N, Zeng GZ, Yin JL and Bian ZX: Artesunate activates the ATF4-CHOP-CHAC1 pathway and affects ferroptosis in Burkitt's lymphoma. Biochem Biophys Res Commun 519: 533-539, 2019.

43. Tang HM and Tang HL: Cell recovery by reversal of ferroptosis. Biol Open 8: bio043182, 2019.

c) (†) $\ominus$ This work is licensed under a Creative Commons EY NC ND Attribution-NonCommercial-NoDerivatives 4.0 International (CC BY-NC-ND 4.0) License. 